1992

\title{
Ethnomethodology, Psychosocial Measures, and Inhalant Abuse Research
}

Joseph E. Trimble, PhD

Western Washington University, joseph.trimble@wwu.edu

Follow this and additional works at: https://_cedar.wwu.edu/psychology_facpubs

Part of the Multicultural Psychology Commons

\section{Recommended Citation}

Trimble, PhD, Joseph E., "Ethnomethodology, Psychosocial Measures, and Inhalant Abuse Research" (1992). Psychology Faculty and Staff Publications. 5.

https://cedar.wwu.edu/psychology_facpubs/5 


\title{
Ethnomethodology, \\ Psychosocial Measures, and Inhalant Abuse Research
}

\author{
Joseph E. Trimble
}

In their chapter, Oetting and Webb set out two fundamental and somewhat ambitious objectives: (1) to assess and describe the psychosocial correlates of inhalant use and (2) to recommend a long-term series of highly focused research projects. In this comprehensive chapter, two basic themes subsume the actual thrust of his work. Specifically, Oetting and Webb emphasize the science of inhalant abuse research and, to a lesser extent, tantalize the reader with their emphasis on the role that culture and ethnicity play in the research. The isolation of these two salient themes stimulated me to focus my comments on selected psychosocial issues and the ethnomethodological ${ }^{1}$ flavor of their writing.

Oetting and Webb, in laying out a progressive series of social and behavioral science methodological approaches, remain somewhat faithful to their profession. They believe that psychology is a natural science and subscribe to the

\footnotetext{
${ }^{1}$ The term "ethnomethodology" was first coined by Garfinkel (1967) and refers to the study of the way ordinary people go about their daily lives. In this chapter, the term has broader implications and broadens the meaning to include the study of culture from an interdisciplinary perspective.
} 
notion that the unit of analysis is the generalized individual. Unlike most of their colleagues, their perspectives are not locked in a segregationist ideology where culture is not viewed as a variable worthy of consideration (Pepitone 1987). Oetting and Webb are not steadfast in keeping to the fundamental guideposts that limit most investigators in the science of the social and behavioral science disciplines. For them culture is an important variable, especially in terms of the methods one chooses to use and the importance of the unique lifeways and thoughtways of the units of analysis. So it is no surprise that they recommend ethnographic approaches and devote space describing inhalant use among different ethnic groups.

Oetting and Webb jump right into the fray of the arguments surrounding appropriate substance abuse research models. They propose a model bounded by methodological and psychosocial dimensions. As intended, the model prompts close scrutiny and begs for analysis. The bait is not easily ignored. The methodological dimension follows a linear progression marked by presumed increasing scientific rigor. They note that scientific inquiry begins with naturalistic observations and ends with treatment research. The progressive dimension resembles the levels of analysis perspective introduced by the sociologist Talcott Parsons (1960) and advanced by the social psychologists Sherif and Sherif (1969).

The methodological dimension prompts me to make a few pointed suggestions. First, I would argue that the ethnographic method should immediately follow naturalistic observation; epidemiological procedures tend to be more quantitative, while ethnography and naturalistic observation lean more to the use of qualitative procedures. In fact, the emphasis a researcher places on quantitative and qualitative procedures fluctuates immensely as one progresses through various levels of research rigor. Second, Oetting and Webb's model can be readily expanded and might be made more useful since each method can be subcategorized into a variety of alternatives. Experimental research, for example, can be subdivided into quasiexperimental studies; this level of inquiry can be further divided into field studies, natural group experiments, etc. Third, there doesn't appear to be room in the scheme for economic, historical, physiological, and psychopharmacological methods. Oetting and Webb might argue that they are preparing a model for psychosocial research, but we cannot lose sight of the obvious fact that psychoactive substances are chemical compounds that interact with the human body in varied and complex ways that indeed influence consciousness. And we know 
all too well that each individual reacts to the substances in a multitude of ways influenced by the history of usage, dosage levels, composition (especially for solvents) of the drugs, metabolic rates, and the availability of the substance. Methods are available to isolate the above listed variables. Without these additional methods of inquiry, the methodological dimension is incomplete and represents only a partial list of those methods necessary to comprehensively understand and predict the dynamic effects of drug use and abuse. Indeed, by including the methodological dimension as a psychosocial method, they omit other important aspects of human behavior.

Unfortunately, the three-part psychosocial dimension consisting of cultural, social, and psychological elements prompts even more stringent criticism. The social element presents very few problems and will not be discussed. The relationship of the psychological element is a bit more confusing. Do Oetting and Webb really mean the individual? Where is there room for the interactive roles of learning and genetics? What role does the findings of sociobiologists play in the psychological (or individual) element? Should the element be subdivided into more refined categories to include personality, psychopathology, and development?

The third element, culture, arouses the most concern. Oetting and Webb's discussion of culture is somewhat inaccurate and redundant. The social and psychological elements in the model, in themselves, are not acultural. In the discussion on culture, Oetting and Webb place the construct in an abstract domain; they state, "culture provides a foundation of. .." Culture is a product and a process, not a foundation. Culture is the result of human interaction both at the individual and group level and therefore is inextricably woven in the fabric of all human activity. Furthermore, Oetting and Webb argue that major socialization forces are based on culture - they are not, as culture permeates those forces in every conceivable manner. For this and many other reasons I prefer to use the term "enculturation" in lieu of "socialization" as it is "the aspect of the learning experience which sets (humans) off from other creatures, and by means which initially and in later life (one) achieves competence ... within the limits of a given body of custom" (Herskovits, 1948, p. 39).

The concept of culture is fraught with conjecture. Just a cursive review of its many definitions reveals its complex nature; one can find slightly over 100 attempts to define the seemingly elusive concept. But whatever definition one chooses, social and psychological processes will somehow be embedded in its 
framework; that is, both processes will be implied by the definition or they will be stated directly.

Oetting and Webb's separate listing of culture also raises the inevitable research question: What research variable status should culture be given? Eckensberger (1979) pointed out that much of the early work in cross-cultural psychology treated culture as an antecedent and consequently provided no strategy for the interpretation of culture as a product of human action and behavioral change. Segall (1983) argues that social and behavioral researchers, rather than strive to comprehend culture, should "identify the various lower-order factors in the natural and man-made environments of humans which influence their behavior in a reliable manner" (p. 127). Hence, Segall believes that "culture cannot be one of these independent variables" ( $\mathrm{p}$. 127). Instead they suggest that it can be given the status of an overarching independent variable. Still others argue that the products of culture should be dependent variables, and, when that occurs, all sorts of measurement, sampling, procedural, and conceptual problems arise owing to a society's distinctive lifeways and thoughtways.

The psychosocial model put forth by Oetting and Webb is, however, appropriate for use in outlining a psychosocial research agenda. Because of the psychosocial domain, the model is somewhat limited in its inclusiveness. Huba, Wingard, and Bentler (1980) proposed a drug use framework that is a bit more comprehensive than that proposed by Oetting and Webb. The model is divided along four progressive dimensions, viz. biological, intrapersonal, interpersonal, and sociocultural. Linkages are established between various research element domains showing the interrelatedness of factors that summarily influence drug use. Oetting and Webb's measurement domains could be added to those of Huba, Wingard, and Bentler (1980) to add some guidance for the researcher.

While I have a partial fondness for comprehensive, ecologically grounded models, they do present horrendous research problems. Drug abuse researchers have been struggling with isolating causal relationships, contingencies, and correlates for decades. Some of the findings make sense; others are short lived as new, contradictory findings introduce alternative explanations. Comprehensive models like those proffered by Oetting and Webb and others are pushing researchers into using a potpourri of variables. We struggle to balance the precious time we have with our respondents and the number of 
questionnaire items we can conceivably wedge in - each item or set of items is intended to give clarity to a variable and in some research efforts they end up as a latent variable in an intricate web of causal vectors in some recursive path model. As the causal vectors increase, so do our attempts to establish those significant paths that best predict drug use - in this instance inhalant abuse. After carefully considering all of the research questions and the model, Moncher et al. (1990) ended up with a questionnaire containing well over 200 items. The questionnaire, by prearrangement with our sampling units, could only be administered in a 60-minute session. We had to reduce the number of items, eliminate scales and variables, and thus erode the pretest reliability of some of our scales. The painful process of item reduction had to be reflected against our research agenda, commitments to research sponsors, and the integrity of our scientific mission. Some critics would argue that any predictive model we generated from our results would be only partially valid since there are many potent sources of causality that could not be included in our study. For example, of necessity we were obligated to ask a series of questions about polydrug usage - so for each drug (e.g., LSD, heroin, smokeless tobacco, crack, cocaine, marijuana) we had to repeat a series of interrelated questions that consumed about 60 percent of our questionnaire. Imagine a drug abuse study involving just one ethnic group where the researchers are driven to be inclusive of the many plausible array of interactive variables conducted with a few of the methods recommended by Oetting and Webb. All things considered, the effort could be enormous.

Few youths experiment with single psychoactive substances such as inhalants (e.g., solvents). Moreover, youths who integrate drugs into their lifestyle, however vigorously, usually are using other substances. Attempting single drug studies can obfuscate the use patterns and the accompanying vicissitudes of other drug use and argues against single method approaches to studying drug use. Certainly such isolated studies have merit and are worthy of consideration. As any drug researcher well knows, drug use patterns are not static phenomena. A temporal and historical factor intrudes on the drug use process that, to a large extent, is molded by changing attitudes, morals, values, drug availability, economics, and variable social sanctions. In a word, most drug studies are time bound. What might have been useful data two decades ago may only have an ounce of relevance for the last decade of the 20th century. To prevent our results from wearing out, do we wear out ourselves and our respondents by designing massive data mining ventures to accumulate and generate as much information as possible? 
Oetting and Webb's chapter, especially the well written and thorough literature review, raises even more painful questions. A number of themes and findings could be isolated for further discussion; in keeping with the theme of this reply, emphasis will be directed toward the ethnic and cultural results.

Oetting and Webb's summary of cross-cultural inhalant abuse findings can be reflected against our review of the ethnic-minority drug abuse literature. Specifically: (1) drug abuse is presented as more of a problem for nonwhite ethnic groups; (2) these ethnics become users and abusers in response to the prevailing disorganization of the social climate; and (3) the study of drug abuse is only appropriate when comparing use and abuse patterns of differen ethnic groups with whites. Consequently other ethnic groups, especially blacks, are "overrepresented," leading to the unfortunate conclusion that these groups have greater drug use problems than whites (Trimble and Bolek 1989). Moreover, Oetting and Webb's review and summary also echoes the review findings of Austin, Johnson, Carroll, and Lettieri (1977) of 13 years ago. Austin and colleagues maintained ethnic drug abuse research lacked any cogent theory to explain the results, that interpretations and theoretical generalizations were not convincing, and that the dimensions of the problem were still not well understood. The two sets of summary statements are not an indictment of Oetting and Webb's review; after all they merely organized and reported what was available to him. Instead, we have a series of psychosocial inhalant abuse findings that actually present more questions than answers and thus we have large gaps in our understanding of the problem.

One of the problems identified by Oetting and Webb concerns the near absence of studies attempting to isolate the correlates between ethnic and cultural identification of minority youth and inhalant use patterns. Oetting and Webb do summarize their own work on the identity-use relationship found in their work among American Indians. By their own admission the results are both mixed and highly complex. Part of the problem involves the psychometric characteristics of ethnic identification measures and the fact that most ethnic-minority drug abuse studies fail to assess the levels of ethnic identity of respondent populations.

More often than not, drug abuse researchers seeking ethnic and cultural explanations select respondents as though they share a common modal understanding of their own ethnicity and nationalistic identification. Oetting and Webb's review of the literature bears out this assumption, as does the field of 
ethnic minority substance abuse research. What we find are studies focusing on American Indians (or Native Americans), Asian and Pacific Americans, Blacks, Mexican Americans, Puerto Ricans, and even studies involving "Hispanics" (Trimble 1991). For a vast majority of the studies descriptions of ethnics tend to rely on the use of broad ethnic glosses, superficial, almost vacuous, categories that serve only to separate one group from another. Use of such glosses gives little or no sense of the richness of cultural variations within these groups, much less the existence of numerous subgroups characterized by distinct lifeways and thoughtways. Forbes (1990) maintains that use of broad ethnic designations in any form is insulting. Heath (1987) offers a more strident observation: "The insult arises from having to deal with someone else with the authority and aggressiveness to assault one's own ability to be one's self" (p. 48). "Even though such gross categories bear no relation to the reference groups with which people normally identify themselves," they claim, "one might expect them at least to yield useful statistics at the national level. Unfortunately, they are utterly useless in that respect as well" (p. 106). Use of ethnic glosses to conduct ethnic-comparative or ethnic-specific studies is not only insulting, it is very poor science. Consider the implications for external validity and replication studies (of which there are very few indeed in the drug abuse field).

So how do we assess ethnic-identity and marginality? The latter is a related construct that Oetting and Webb identified as a major theme. Most importantly, respondents should be given the opportunity to self-identify, a point emphasized by Trimble (1991) and Forbes (1990). Measuring ethnicity, however, "is not a simple all or nothing proposition. Researchers have long recognized that a person's level or intensity of identification with a particular ethnicity can vary from a weak-nominal association to a strong-committed association" (Smith 1980, p. 79).

Admittedly somewhat contradictorily in light of previous comments, I am recommending that ethnic identification be the mainstay of all studies involving ethnic groups. Self-identification by itself is not sufficient since it creates yet another variation of the ethnic gloss. Measures (which mean more questionnaire items and therefore more time) should tap into the depth and subtle layers of one's ethnicity. As a possibility I suggest that the tripartite measurement domains depicted in figure 1 serve as a guide. As currently 
Figure 1. Ethnic identification measurement domains

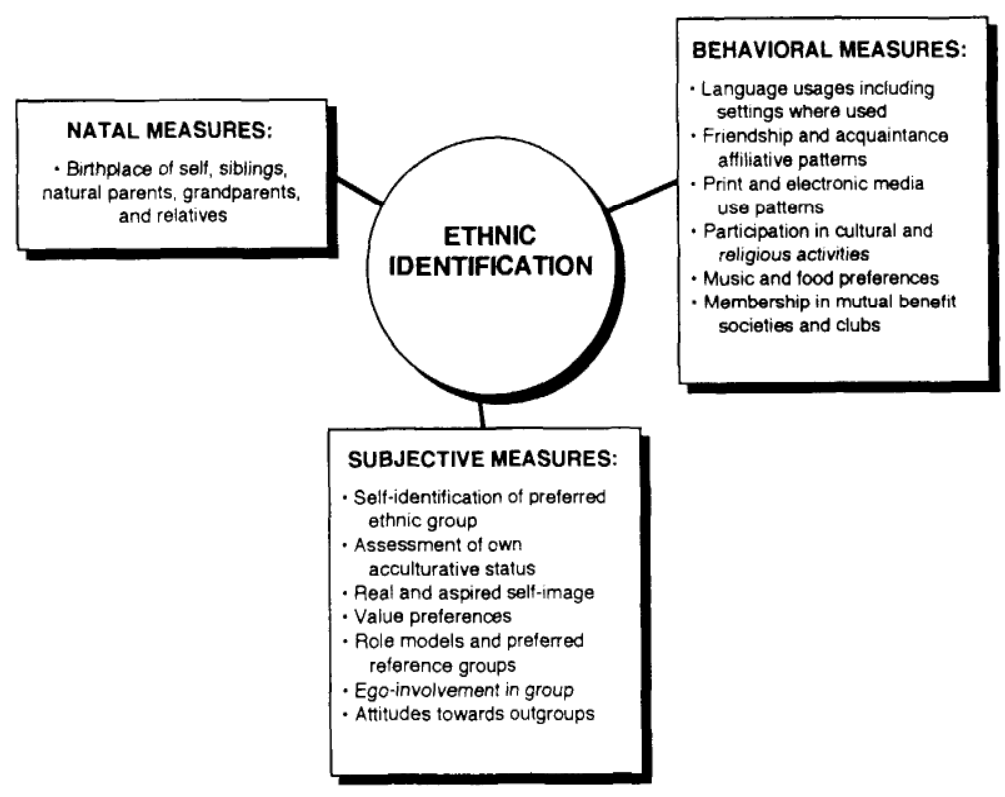


conceived, ethnic identity consists of the subjective, behavioral, and natal domains. Suitable variables are listed beneath each domain that when aggregated across the measures would yield a unified index. Respondent indices most likely would vary across a continuum. Such variation permits numerous statistical manipulations not otherwise available to the researcher who uses ethnic glosses. Moreover, strong, reliable ethnic identification measures would increase external validity, offering the field a more valid array of findings (Trimble 1991).

Marginality, while an interesting concept, has not received the benefit of a good deal of research. That is, there are very few scales to assess it. In part, marginality is really a component of the acculturative process as determined by social class, socioeconomic status, ethnicity, and a few other salient but distinguishing characteristics. Oetting and Webb's claims that inhalant users are found among the marginal ones at any age and in any group and "are marginal in society" need more substantiation. Gist and Wright (1973) maintain that groups are marginal when: (1) members do not ordinarily qualify for admission into another group with which it is more or less closely associated; (2) these groups differ significantly in the nature of their cultural or racial heritage; and (3) between them there is limited cultural exchange or social interaction. Are there empirical data available to demonstrate indeed that inhalant users are marginal? Are some users more marginal than others? Can some form of intervention strategy be devised to chip away at the social and psychological factors that contribute to marginality? Are the users perennially marginal or do they sense their marginality when they feel excluded from groups to which they aspire to affiliate? Inhalant users may be at the edge, but it seems to me that some sense of belonging occurs through peer clustering and the correspondent use of inhalants and presumably other drugs. Scales should be developed to assess the marginality hypothesis. The continued use of the term without adequate substantiation provides the field with a thin excuse to explain away the problem.

Finally, I want to draw some attention to the notion that inhalant and polydrug users have something wrong with their sense of self. Many lay people and some researchers seem to be firmly committed to the idea that when youths use drugs, there one will find a distorted, negatively imbalanced relationship between ideal self and real self. Oetting and Webb did not find many studies examining the self-ideal notion among inhalant users. The ongoing drug use research being conducted at the National Center for American 
Indian and Alaska Native Mental Health Research, which was conducted at an American Indian boarding school, yielded no significant statistical relationship for a measure of self-esteem and drug use (S. Manson, personal communication). In fact, self-measures are weak predictors in their regression analyses. Moreover, Moncher et al. (1990) found similar results among Indian youths in the State of Washington. Other researchers, too, have and continue to get mixed results in their studies relating self-perception or regard to the causes, prevention, and intervention of drug use. There may be a relationship. However, if we are going to continue to explore its possibilities, a good deal of solid psychometric research should be conducted. First, most drug researchers do not use the same measures of self. What is most often used are variations of Rosenberg's self-esteem scale. Hence, researchers are encouraged to settle on using comparable measures of self. Second, the concept of self is extraordinarily complex. Therefore, research is needed to isolate what self domain (e.g., esteem, understanding, control, alienation, mattering, acceptance, efficacy) is most associated with drug use. Third, we can no longer accept drug-related self-study results that are based on very short four to eight item scales. Whether the results are significant or not, use of small item scales tends to obscure observed relationships and general overall assumptions about the effectiveness of a prevention or intervention modality, and, in the main, can lead to gross exaggerations of statistically derived mean effects, especially if one has large samples. And, finally, a concerted, concentrated effort is needed to explore the theoretical relationship between the sense of self and what drug use really does to the general personality makeup of users. I have never quite understood why someone who ostensibly feels good about one's self would use a consciousness altering substance that would enhance that feeling.

\section{References}

Austin, G.A.; Johnson, B.D.; Carroll, E.E.; and Lettieri, D.J., eds. Drugs and Minorities. Research Issue 21. Rockville, MD: National Institute on Drug Abuse, 1977.

Eckensberger, L.H. A metamethodological evaluation of psychological theories from a cross-cultural perspective. In: Eckensberger, L.H.; Lonner, W.J.; and Poortinga, Y.H., eds. Cross-Cultural Contributions to Psychology. Lisse: Swets and Zeitlinger, 1979. pp. 255-275. 
Forbes, J.D. The manipulation of race, caste and identity: Classifying Afroamericans, Native Americans and red-black people. J Ethnic Studies 17(4):1-51, 1990.

Garfinkel, H. Studies in Ethnomethodology. Englewood Cliffs, NJ: PrenticeHall, 1967.

Gist, N. and Wright, R. Marginality and Identity. Leiden: Brill, 1973.

Heath, D.B. Anthropology and alcohol studies: Current issues. Ann Rev Anthropol 16:99-120, 1987.

Hcrskovits, M.J. Man and His Work: The Science Of Cultural Anthropology. New York, NY: Knopf, 1948.

Huba, G.J., Wingard, J.A., and Bentler, P. Framework for an interactive theory of drug use. In: Lettieri, D.J.; Sayers, M.; and Pearson, H.W., eds. Theories on Drug Abuse: Selected Contemporary Perspectives. Research Monograph 30. Rockville, MD: National Institute on Drug Abuse, 1980. pp. 95-101.

Moncher, M.; Holden, G.; and Trimble, J. Substance abuse among NativeAmerican youth. $J$ Consult Clin Psychol 58:400-415, 1990.

Parsons, T. Structure and Process in Modem Societies. New York: Free Press, 1060 .

Pepitone, A. The role of culture in theories of social psychology. In: Kagitcibasi, C., ed. Growth and Progress in Cross-Cultural Psychology. Amsterdam: Swets \& Zeitlinger, 1987. pp. 12-21.

Segall, M. On the search for the independent variable in cross-cultural psychology. In: Irvine, S.H., and Berry, J.W., eds. Human Assessment and Cultural Factors. New York: Plenum, 1983. pp. 127-137.

Sherif, M., and Sherif, C. Social Psychology. New York: Harper and Row, 1969.

Smith, T.W. Ethnic measurement and identification. Ethnicity 7:78-95, 1980.

Trimble, J.E. Ethnic specification, validation prospects and the future of substance abuse research. Int $J$ Addict, 1991. 
Trimble, J.E. and Bolek, C.S. "Ethnic Minority Substance Abuse Research Perspectives: A Literature Review With Commentary." Paper presented at a conference sponsored by the National Institute on Drug Abuse on the topic of conducting cross-cultural substance abuse research, Rockville, MD, May 1989.

\section{Author}

Joseph E. Trimble, Ph.D

Center for Cross-Cultural Research

Department of Psychology

Western Washington University

Bellingham, WA 96225 